\title{
Simultaneous Prediction of Bendability and Deep Drawability Using Orientation Distribution Function for Aluminum Alloy Sheets ${ }^{* 1}$
}

\author{
Hirofumi Inoue ${ }^{* 2}$ \\ Department of Materials Science, Graduate School of Engineering, Osaka Prefecture University, Sakai 599-8531, Japan
}

Sheet metal formability is generally affected by crystallographic texture. In particular, bendability and deep-drawability of aluminum and its alloys are closely related to the recrystallization texture of the rolled sheets. It is necessary to quantitatively predict them from a viewpoint of texture control. This paper described a method for simultaneous prediction of both the bendability and the deep-drawability on the basis of the average Taylor factor as a polycrystal calculated by using an orientation distribution function. The normalized Taylor factor $\left(M_{\mathrm{n}^{-}}\right.$ value) and the $r$-value were used as measures of bendability and deep-drawability, respectively. The predicted results from ideal orientations demonstrated that $\{001\}<100>$ orientation had excellent bendability and poor deep-drawability, whereas $\{111\}<110>$ orientation had poor bendability and excellent deep-drawability. The predicted results for some aluminum alloys suggested that conventional cold-rolled and annealed sheets would be favorable to bendability, and the addition of asymmetric warm rolling after cold rolling would lead to improved deep-drawability. [doi:10.2320/matertrans.L-M2017857]

(Received November 30, 2017; Accepted December 10, 2017; Published February 2, 2018)

Keywords: aluminum alloy, sheet metal formability, orientation distribution function, average Taylor factor, $r$-value

\section{Introduction}

In recent years, aluminum alloy sheets are used for the bodies of some automobiles to reduce the weight. It is known that the formability of sheet steel commonly used as the body consists of four basic deformation components: deep drawability, stretch formability, stretch-flange formability and bendability ${ }^{1)}$. Among them, deep drawability ${ }^{2)}$ and bendability $^{3)}$ are strongly affected by crystallographic orientations and consequently depend on textures of polycrystalline metal sheets.

As for the body of an automobile, since outer panels of the hood, trunk-lid and doors require hemming which corresponds to $180^{\circ}$-bending, good bendability is necessary for aluminum alloy sheets employed as the outer panels. Takeda et $a l .{ }^{3)}$ showed that there was a negative correlation between the bendability on a plane strain condition and the Taylor factor on the basis of experimental results of Al-Mg-Si alloy single crystals with various orientations, although the data had random variation. Similarly, Kaneko and Eguchi ${ }^{4)}$ examined the effect of crystallographic texture on the bendability of $\mathrm{Cu}-\mathrm{Ni}-\mathrm{Si}$ alloy sheets with various textures, and found a positive correlation between the minimum bend radius and the average Taylor factor which meant the average value of the Taylor factor as a polycrystalline sample. Thus it is clear that bendability is correlated with the Taylor factor and the orientation with a lower Taylor factor results in better bendability. Since the properties of polycrystalline materials are expressed by the average values of those of individual grains, the author ${ }^{6)}$ recently proposed a method for predicting the bendability from the average Taylor factor of polycrystalline face-centered cubic metal sheets calculated using an orientation distribution function $(\mathrm{ODF})^{5)}$ which expressed a volume fraction of grain orientation.

When considering sheet metal formability in the body of

\footnotetext{
${ }^{* 1}$ This Paper was Originally Published in Japanese in J. JILM 66 (2016) 582-588.

${ }^{* 2}$ Corresponding author, E-mail: inoue@mtr.osakafu-u.ac.jp
}

automobiles, sheet materials with good deep-drawability are necessary for door inner panels and fenders which require forming the sheets into complex components. Lankford value $(r \text {-value })^{7)}$ as a measure of deep drawability, however, has a considerably low value in normally rolled and annealed aluminum alloy sheets as compared to in steel sheets. Therefore the $r$-value which strongly depends on orientation should be improved through texture control ${ }^{2)}$. Recently the authors succeeded the formation of recrystallization texture consisting mainly of $\{111\}<110>$ orientations favorable for improving the $r$-value as a main texture component ${ }^{8-10)}$. Fine texture control requires predicting in-plane anisotropy of the $r$-value with high accuracy. A method for predicting the $r$-value based on the average Taylor factor calculated by using ODF is very useful ${ }^{2,5,11,12)}$.

In the present study, the average Taylor factor of a polycrystalline material was calculated through texture analysis by ODF. Then the anisotropic in-plane features of the normalized Taylor factor and the $r$-value as respective criteria of bendability and deep drawability were predicted simultaneously. In addition, the normalized Taylor factor and the $r$-value were calculated by using real recrystallization texture data for aluminum alloy sheets ${ }^{9,13)}$.

\section{Analytical and Experimental Methods}

Tensile deformation is first explained as the basics of bending deformation and the $r$-value analysis.

\subsection{Calculation of Taylor factor in tensile deformation}

In tensile deformation of a sheet material, the longitudinal, transverse and thickness directions are defined as the $X$-, $Y$ - and $Z$-axes, respectively. Here we assume that the volume element with unit length is macroscopically elongated by $\mathrm{d} \varepsilon$ in the $X$-direction and is contracted by $q \mathrm{~d} \varepsilon$ and $(1-q) \mathrm{d} \varepsilon$ in the $Y$ - and $Z$-directions, respectively, that is, the condition of constant volume, $\mathrm{d} \varepsilon_{x}+\mathrm{d} \varepsilon_{y}+\mathrm{d} \varepsilon_{z}=0$ is satisfied under macroscopically uniform plastic deformation. In this case, the strain tensor $E^{\mathrm{S}}$ is expressed by the following equation. 


$$
E^{\mathrm{S}}=\mathrm{d} \varepsilon \cdot\left[\begin{array}{ccc}
1 & 0 & 0 \\
0 & -q & 0 \\
0 & 0 & -(1-q)
\end{array}\right]
$$

Thus the $q$-value represents a ratio of transverse to longitudinal strain $(0 \leq q \leq 1)$.

Now we assume that individual grains are subjected to homogeneous strain identical with macroscopic tensile strain to satisfy the continuity in strain at grain boundaries. In order to view the strain condition of eq. (1) in the crystal coordinate system, $X^{\mathrm{C}}=[100], Y^{\mathrm{C}}=[010]$ and $Z^{\mathrm{C}}=[001]$, the coordinate transformation is performed from the tensile specimen coordinate system $X-Y-Z$. There are five independent strain components in the coordinate-transformed strain tensor in view of both the symmetry of strain and the condition of constant volume. Thus at least five active slip systems are necessary for plastic deformation in each grain. The principle of the minimum work is here introduced to determine the combination of five active slip systems. If the critical resolved shear stress (CRSS) of $\{111\}<110>$ glide systems is $\tau_{c}$ and the shear strain of a given glide system is represented by $\mathrm{d} \gamma_{n}$, then the plastic work by slip deformation is expressed by the following equation.

$$
\mathrm{d} W=\tau_{c} \sum_{n=1}^{5}\left|\mathrm{~d} \gamma_{n}\right|
$$

Hence the combination of glide systems in which $\mathrm{d} W$ is minimum, i.e. the sum of absolute value of shear strain is minimum, is selected as five active slip systems. In the case of tensile deformation, the plastic work by external stress is expressed by $\sigma \cdot \mathrm{d} \varepsilon$ and is equal to the right side of eq. (2). Thus the Taylor factor $M$, which is a function of crystallographic orientation $g$ and strain ratio $q$, is given by the following equation.

$$
M=\frac{\sigma}{\tau_{c}}=\min \left(\sum_{n=1}^{5}\left|\mathrm{~d} \gamma_{n}\right|\right) / \mathrm{d} \varepsilon
$$

Calculating the Taylor factor $M(q, g)$ at a given $q$-value, the relation between $g$ and $M(q, g)$ is determined. In order to simplify subsequent calculations, we calculate an approximation of $M(q, g)$ in the form of series-expansion by generalized spherical harmonics $T_{l}^{\mu \nu}(g)^{5)}$ which take crystal and sample symmetry into account.

$$
M(q, g)=\sum_{l=0}^{L} \sum_{\mu=1}^{M(l)} \sum_{v=1}^{N(l)} m_{l}^{\mu v}(q) T_{l}^{\mu v}(g)
$$

In this manner, the coefficients $m_{l}^{\mu \nu}(q)$ can be determined by calculating $M(q, g)$ at an appropriate interval from $q=0$ to $q=1 . q=0$ and $q=1$ correspond to plane strain deformation and $q=0.5$ corresponds to axisymmetric deformation.

In the meantime, the texture in a polycrystalline metal can be quantitatively expressed using an orientation distribution function (ODF) $f(g)^{5)}$ shown in the following equation.

$$
f(g)=\sum_{l=0}^{L} \sum_{\mu=1}^{M(l)} \sum_{v=1}^{N(l)} C_{l}^{\mu v} T_{l}^{\mu v}(g)
$$

The coefficients $C_{l}^{\mu v}$ can be calculated from some pole figures on different crystal planes measured by X-ray diffraction. When the tensile-strain direction is identical with the rolling direction, the mean value of the Taylor factor as the whole of specimen, namely the average Taylor factor, $\bar{M}(q)$, is obtained by taking the weighted average of $M(q, g)$ with $f(g)$ in the whole orientation space.

$$
\bar{M}(q)=\oint M(q, g) f(g) \mathrm{dg}
$$

Using the coefficients $m_{l}^{\mu v}(q)$ and $C_{l}^{\mu v}$ in eq. (4) and eq. (5) respectively, eq. (6) can also be expressed as the following equation.

$$
\bar{M}(q)=\sum_{l=0}^{L} \sum_{\mu=1}^{M(l)} \sum_{v=1}^{N(l)} \frac{m_{l}^{\mu v}(q) C_{l}^{\mu v}}{2 l+1}
$$

If we use the tensile specimen cut along an arbitrary direction to the rolling direction in a sheet, then the average Taylor factor in the specific direction is given by the following equation using the angle $\theta$ to the rolling direction.

$$
\bar{M}(q, \theta)=\sum_{l=0}^{L} \sum_{\mu=1}^{M(l)} \sum_{v=1}^{N(l)} \frac{m_{l}^{\mu v}(q) C_{l}^{\mu \nu} \cos 2(v-1) \theta}{2 l+1}
$$

Therefore, the curve $\bar{M}(q, \theta)$ as a function of $\theta$ under a fixed $q$-value represents an in-plane variation of the average Taylor factor in a polycrystalline sheet material.

\subsection{Prediction of bendability}

It is known that crack initiation during bending deformation is related to the formation of shear bands and texture strongly affects the shear bands formation ${ }^{3,4,14)}$. The CRSS is identical in face-centered cubic metals with only $\{111\}<110>$ glide systems at room temperature. Therefore, assuming that as the Taylor factor lowers, i.e. as the total amount of glides becomes smaller, it is more difficult for shear bands to form, the average Taylor factor of a polycrystalline material was calculated from the ODF, and then utilized for predicting bendability.

In bending deformation, the $q$-value of eq. (8) corresponds to a ratio of compressive strain along the bending axis to tensile strain along the bending direction on the side subjected to tensile stress. When an outer side (a convex side) in bending deformation satisfies the condition of plane strain tension, the $q$-value equals zero. In fact the $q$-value is considered to be a little larger than zero on the sheet surface subjected to the maximum tensile stress when the width of a bending specimen is relatively small. An in-plane variation of the average Taylor factor can be given by the graph showing a relationship between $\theta$ and $\bar{M}(q, \theta)$ under a fixed $q$-value in eq. (8). To quantitatively relate the average Taylor factor to bendability, we further calculate the average Taylor factor for a textured sheet material $\bar{M}(q, \theta)$ divided by the Taylor factor for a randomly oriented sample (i.e. by the coefficient of the first term in eq. (4)) $m_{0}^{11}(q)$, which is defined 
as the normalized Taylor factor (the Mn value) ${ }^{6}$. Therefore the $M_{\mathrm{n}}$ value of 1.0 corresponds to bendability for a randomly oriented sample.

$$
M_{\mathrm{n}}(q, \theta)=\frac{\bar{M}(q, \theta)}{m_{0}^{11}(q)}
$$

In the present study, the $M_{\mathrm{n}}$ value was calculated for $q=$ $0.1^{6)}$ suitable for bendability prediction by setting the interval of angle $\theta$ at $15^{\circ}$.

\subsection{Prediction of deep drawability}

Since the $r$-value is a strain ratio of width and thickness directions in a tensile specimen, the $r$-value can be expressed by the following equation using the $q$-value which corresponds to a strain ratio of width and longitudinal directions.

$$
r=\frac{q}{1-q}
$$

The average Taylor factor $\bar{M}(q, \theta)$ can be calculated using eq. (8). Thus the $r$-value can be determined by calculating the $q$-value which $\bar{M}(q, \theta)$ becomes minimum at a fixed angle of $\theta$. Here $r=1$ (i.e. $q=0.5$ ) means the $r$-value for a randomly oriented sample. If the $q$-value which $\bar{M}(q, \theta)$ becomes minimum is determined at various angles of $\theta$, then an in-plane variation curve of the $r$-value is obtained. In fact the $r$-value was calculated at a constant angle interval of $15^{\circ}$. To compare the calculated results of all aluminum alloys, the full constraints model suitable for the $r$-value prediction of Al-Mg-Si alloy sheets ${ }^{2}$ was used in the present study (See eq. (1)). As mentioned above, it is possible to predict bendability and deep drawability simultaneously by calculating the average Taylor factor $\bar{M}(q, \theta)$ using ODF.

\subsection{Prediction of the $M_{\mathrm{n}}$ value and the $r$-value for ideal orientations}

A single texture component, that is a family of preferred orientations consisting of crystallographically equivalent variants, was artificially generated, which was expressed by the ODF with Gaussian spread ${ }^{5)}$ of $20^{\circ}$. The width of Gaussian spread was set in view of orientation density compatible to that of a real texture. It should be noted that one texture component does not mean a strict orientation $(h k l)[u v w]$ of a single crystal but consists of all the equivalent orientations $\{h k l\}<u v w>$ of a polycrystal in terms of orthogonal sample symmetry of a rolled sheet. The average Taylor factor $\bar{M}(q, \theta)$ was calculated using eq. (8) from the coefficients $C_{l}^{\mu \nu}$ of the ODF for a given texture component $\{h k l\}<u v w>$. Then the $M_{\mathrm{n}}$ value and the $r$-value were determined by the above methods.

\subsection{Prediction of the $M_{\mathrm{n}}$ value and the $r$-value for alu- minum alloy sheets}

In addition to a 1050 commercially pure aluminum sheet with $\mathrm{O}$ temper, a 5182 aluminum alloy sheet with $\mathrm{O}$ temper and a 6022 aluminum alloy sheet with T4 temper employed as common samples of the Sectional Meeting on Textures in the Japan Institute Light Metals ${ }^{13)}$, a 6022 aluminum alloy sheet which was $90 \%$ cold rolled, $25 \%$ asymmetrically warm rolled at $473 \mathrm{~K}$ with a roll speed ratio of 1.5 and T4- treated $^{9)}$ was used for texture measurements. The $\{111\}$, $\{100\},\{110\}$ and $\{311\}$ incomplete pole figures of these samples were measured using $\mathrm{CuK} \alpha$ radiation by Schulz reflection method ${ }^{15)}$ in a tilt angle range of $\Phi=15^{\circ}$ to $90^{\circ}$. For the sheet materials employed in the Sectional Meeting on Textures, the pole figures were measured on the sheet surface after lightly removing oxides by chemical etching6). Using ODF analysis by the iterative series-expansion method $^{16)}$ developed by the author, the coefficients $C_{l}^{\mu \nu}$ were calculated from measured incomplete pole figures, and then the $M_{\mathrm{n}}$ value and the $r$-value were determined by the above methods. In the case of the cold rolled and asymmetrically warm rolled A6022-T4 sample, ODF analysis was made using RD pole figures measured for a stacked sheets specimen to examine an average texture through sheet thickness ${ }^{17)}$. Computer program for predicting the $M_{\mathrm{n}}$ value and the $r$-value operates in connection with general-purpose ODF analytical software (Standard $\mathrm{ODF}^{18)}$ ) on Microsoft Windows.

\section{Results and Discussion}

\subsection{In-plane variations of the $M_{\mathrm{n}}$ value and the $r$-value for ideal orientations}

Figure 1(a) shows an ODF of cube orientation $\{001\}<100>$ with Gaussian distribution. Considering the orthogonal sample symmetry, the multiplicity is 4 . Thus the cube orientation consists only of one variant, and the maximum orientation density of the ODF has a high value of 48.5. In-plane variations of the normalized Taylor factor $M_{\mathrm{n}}$ and the $r$-value for the cube orientation calculated at $q=0.1$ are shown in Fig. 1(b). The $M_{\mathrm{n}}$ value shows a very low value of about 0.9 at all angles to the rolling direction. Here the angle $\theta$ means the bending direction perpendicular to a bend axis, which is subjected to tensile or compressive strain during bending deformation. Therefore the bendability of cube orientation can be estimated to be very excellent in any direction. The predicted results for bendability correspond well to experimental results by Takeda et al. ${ }^{3)}$ In contrast, the $r$-value shows a very low value less than 0.1 at $\theta=45^{\circ}$ and the in-plane anisotropy is remarkable. Thus the deep drawability of cube orientation is estimated to be bad.

$\mathrm{S}$ orientations $\{123\}<634>$ (Fig. 2(a)), which consist of four variants symmetrical to the RD and TD axes in pole figures (i.e. a multiplicity of 1) and show the maximum orientation density (11.6) of about quarter value compared with cube orientation, have isotropic $M_{\mathrm{n}}$ values being slightly higher than 1.0 (Fig. 2(b)). The $r$-value shows a higher value than 1.0 around $\theta=45^{\circ}$ in contrast with cube orientation but the in-plane anisotropy is relatively large. Thus it is estimated for $\mathrm{S}$ orientations that the bendability is not good and the deep drawability is not so good.

$\{111\}<110>$ orientations (Fig. 3(a)), which consist of two variants symmetrical to the RD axis in pole figures (i.e. a multiplicity of 2) and show the maximum orientation density (24.7) of about half value compared with cube orientation, have higher $M_{\mathrm{n}}$ values than those of S orientation (Fig. 3(b)). The $r$-value shows a high value more than 1.5 in any direction and similar in-plane anisotropy to that of the $M_{\mathrm{n}}$ value. Thus it is estimated for $\{111\}<110>$ orientations that the 


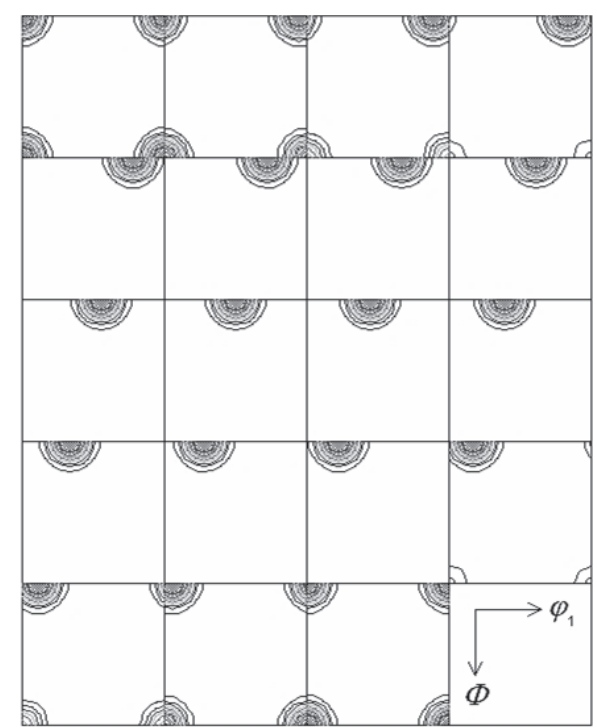

( a )

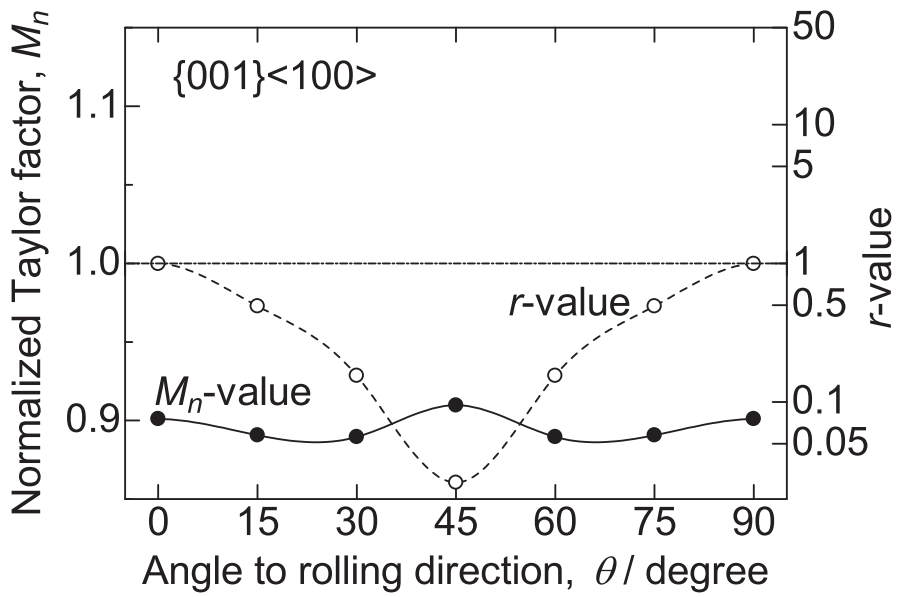

( b )

Fig. 1 (a) Orientation distribution function (ODF) with maximum orientation density of 48.5 for ideal orientation $\{001\}<100>($ Contour levels: 3.0 to 45.0 at a step of 3.0), and (b) in-plane variations of normalized Taylor factor and r-value calculated from the ODF.

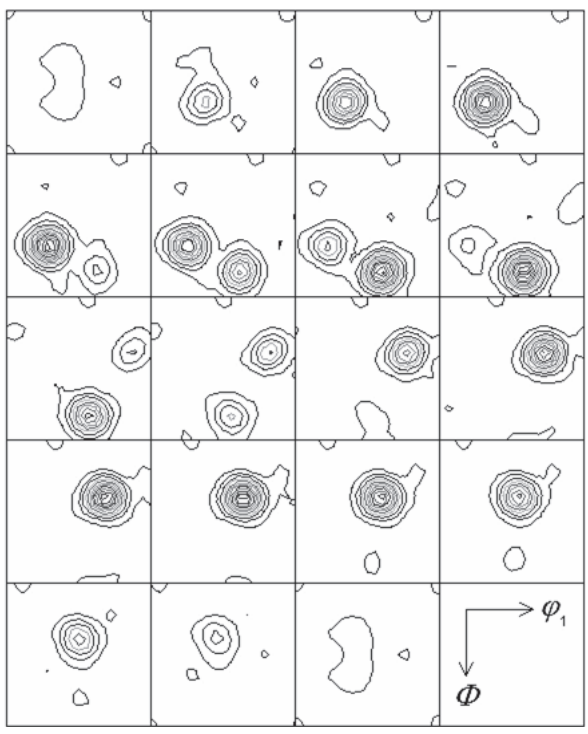

( a )

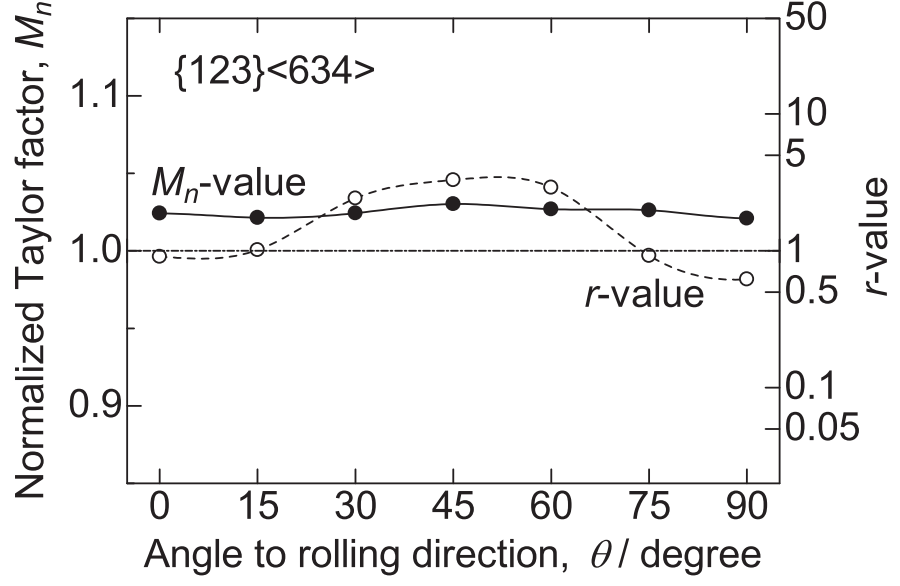

( b )

Fig. 2 (a) Orientation distribution function (ODF) with maximum orientation density of 11.6 for ideal orientation $\{123\}<634>($ Contour levels: 1.0 to 11.0 at a step of 1.0), and (b) in-plane variations of normalized Taylor factor and $r$-value calculated from the ODF.

bendability is not good but the deep drawability is very excellent. The predicted results for deep drawability correspond to experimental results by authors obtained in a sample with $\{111\}<u v w>$ orientation components ${ }^{2)}$.

From the predicted results from these ideal orientations, $\{001\}<100>$ and $\{111\}<110>$ orientations are effective for improving bendability and deep drawability, respectively. It is obvious that texture control suitable for the use is necessary to improve sheet metal formability.

\subsection{In-plane variations of the $M_{\mathrm{n}}$ value and the $r$-value for aluminum alloy sheets}

Figure 4 shows an ODF for the surface area of a 1050 aluminum sheet with $\mathrm{O}$ temper and in-plane variations of the $M_{\mathrm{n}}$ value and the $r$-value calculated from the ODF. Since there is a sharp cube texture including RD-rotated components from cube preferred orientation (an orientation spread along the $\Phi$ axis in the $\varphi_{2}=0^{\circ}$ section), this material has considerably low the $M_{\mathrm{n}}$ value similar to that of cube orientation in Fig. 1 and the $M_{\mathrm{n}}$ value increases slightly with increasing angle $\theta$, resulting from RD-rotated weak components. The $r$-value shows a very low value around $\theta=45^{\circ}$ 


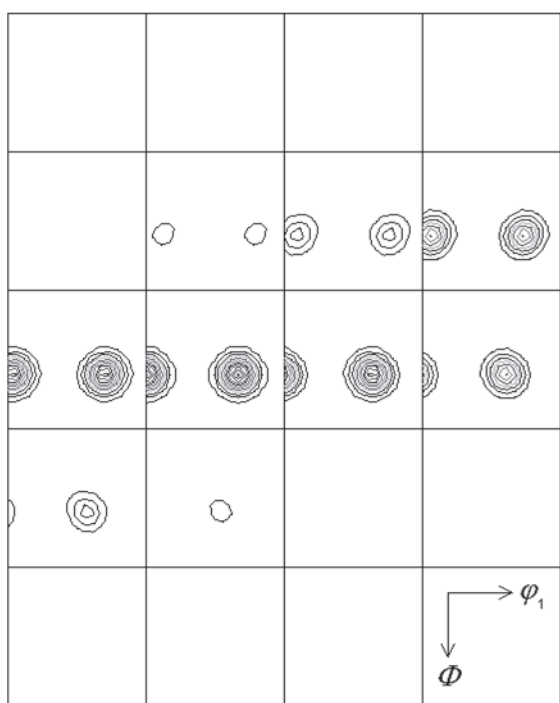

(a)

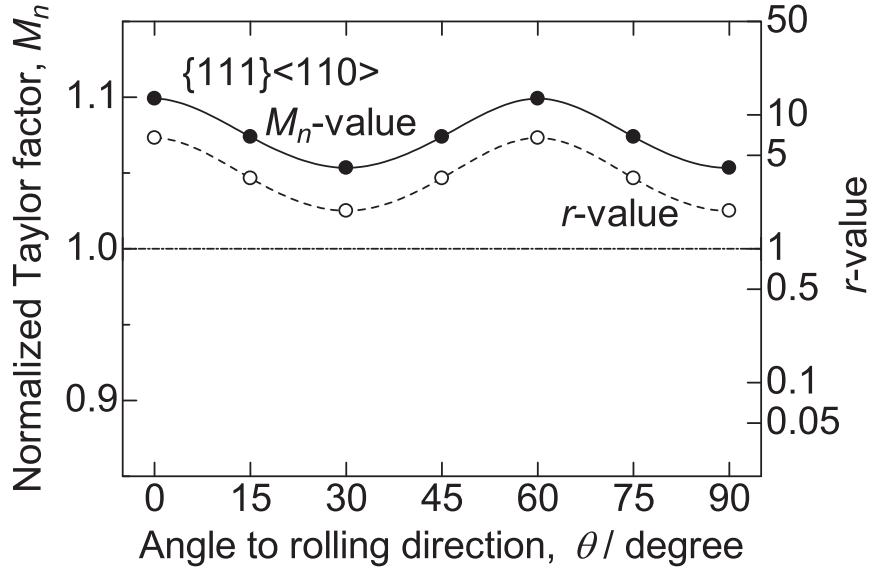

(b)

Fig. 3 (a) Orientation distribution function (ODF) with maximum orientation density of 24.7 for ideal orientation $\{111\}<110>($ Contour levels: 2.0 to 24.0 at a step of 2.0.), and (b) in-plane variations of normalized Taylor factor and $r$-value calculated from the ODF.

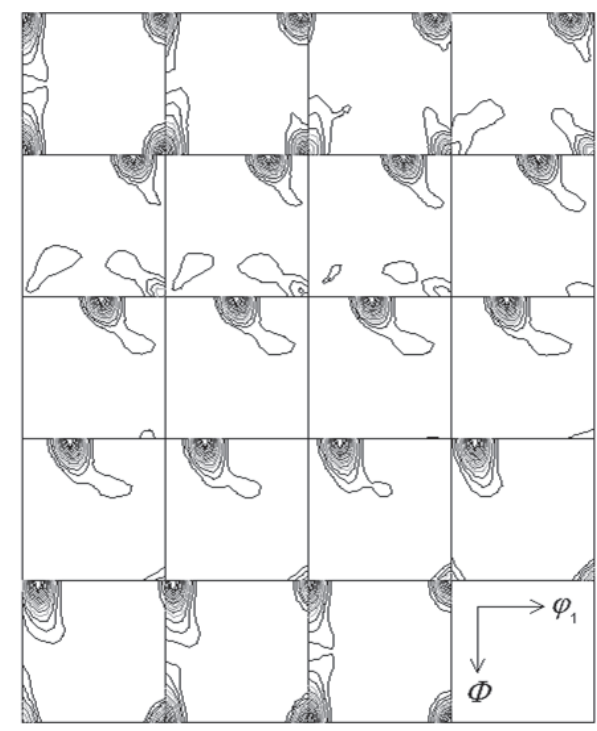

(a)

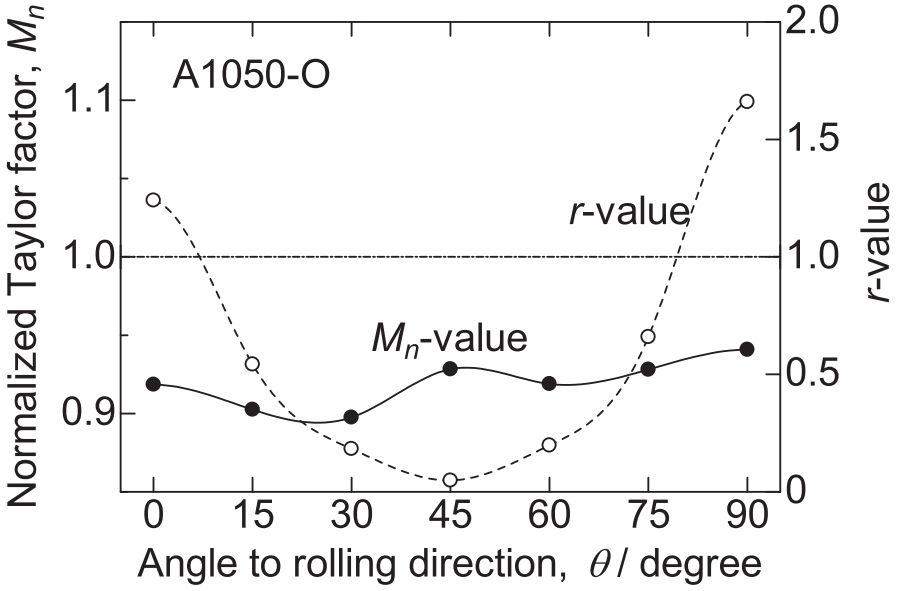

(b)

Fig. 4 (a) Orientation distribution function (ODF) with maximum orientation density of 35.2 for 1050 aluminum sheet with O temper (Contour levels: 2.0 to 30.0 at a step of 2.0), and (b) in-plane variations of normalized Taylor factor and $r$-value calculated from the ODF.

and the in-plane anisotropy is considerably remarkable. Thus it is estimated for A1050-O that the bendability is excellent but the deep drawability is not good.

ODFs for the surface areas of a 5182 aluminum alloy sheet with $\mathrm{O}$ temper and a 6022 aluminum alloy sheet with T4 temper and in-plane variations of the $M_{\mathrm{n}}$ value and the $r$-value calculated from the ODFs are shown in Fig. 5 and Fig. 6, respectively. Since A5182-O has a weak ND-rotated cube orientation of $\{001\}<310>$ as a main component, the $M_{\mathrm{n}}$ value is moderately lower than 1.0 and relatively isotropic $\left(M_{\mathrm{n}}<1.0\right.$ in any direction). Similarly the $r$-value is relatively isotropic and on the whole has a low value of about 0.5 , although it shows a rather high value at $\theta=\sim 45^{\circ}$ In con- trast, A6022-T4 shows large spreads toward ND-rotated cube orientation of $\{001\}<310>$ and RD-rotated cube orientation of $\{011\}<100>$, but has moderately strong cube texture. Therefore the $M_{\mathrm{n}}$ value is relatively low and isotropic. The $r$-value shows the lowest value at $\theta=45^{\circ}$ and on the whole becomes somewhat lower than that of A5182-O. When ignoring the effect of ductility on sheet formability, in view of texture, A6022-T4 is better in bendability than A5182-O, while A5182-O is somewhat better in deep drawability than A6022-T4.

Figure 7 shows the results of a T4-treated material for the 6022 aluminum alloy sheet which was $90 \%$ cold rolled and $25 \%$ asymmetrically warm rolled. A main component of the 


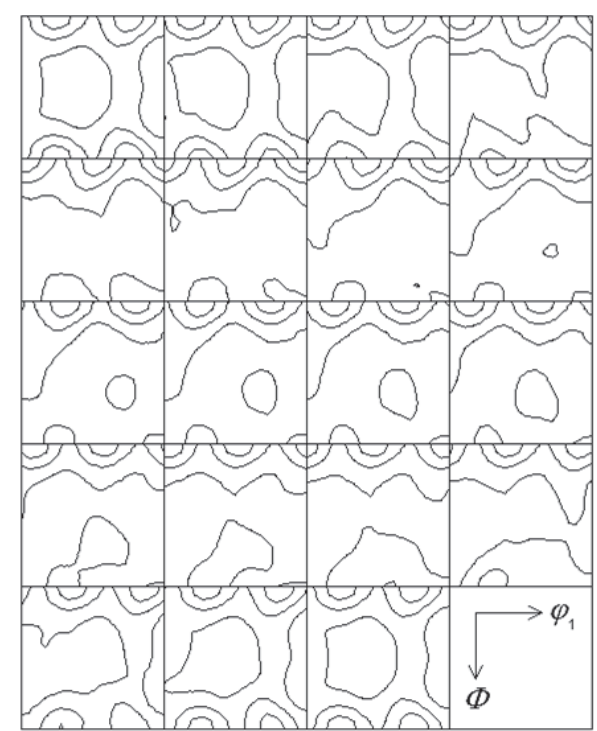

( a )

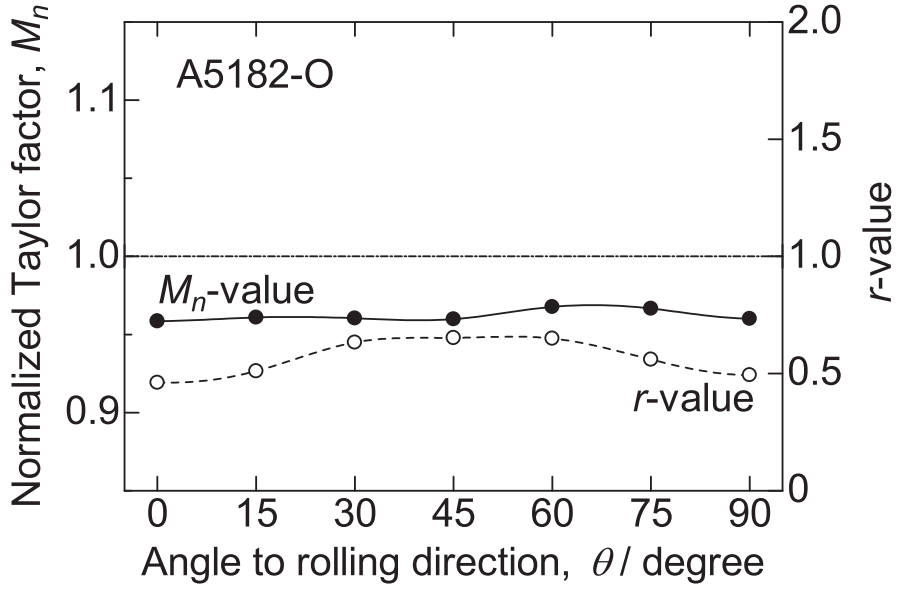

(b)

Fig. 5 (a) Orientation distribution function (ODF) with maximum orientation density of 3.7 for 5182 aluminum alloy sheet with O temper (Contour levels: 1.0 to 3.0 at a step of 1.0), and (b) in-plane variations of normalized Taylor factor and $r$-value calculated from the ODF.

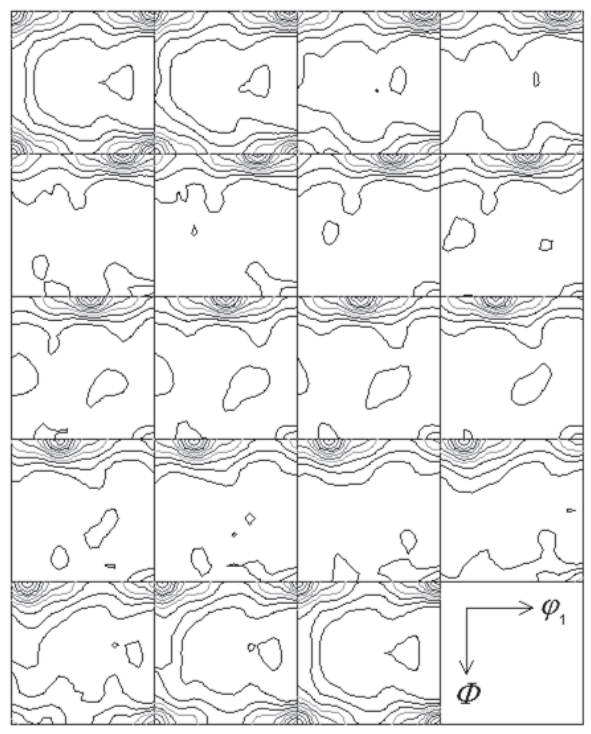

( a )

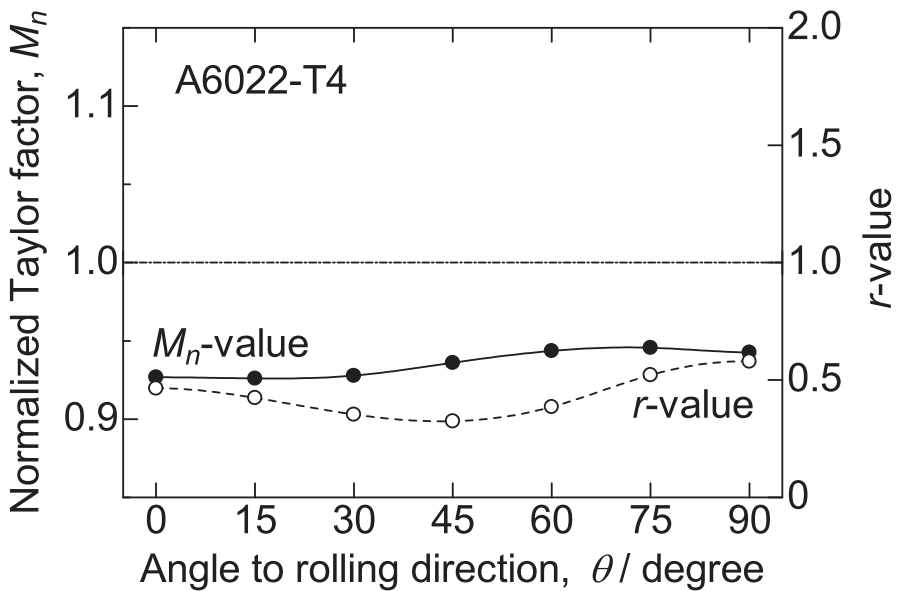

( b )

Fig. 6 (a) Orientation distribution function (ODF) with maximum orientation density of 11.2 for 6022 aluminum alloy sheet with T4 temper after 90\% cold rolling (Contour levels: 1.0 to 11.0 at a step of 1.0), and (b) in-plane variations of normalized Taylor factor and $r$-value calculated from the ODF.

recrystallization texture consists of near $\{111\}<110>$ and cube orientation $\{001\}<100>$ exists as the second component. As inferred from $\{111\}<110>$ orientations of Fig. 3, the $M_{\mathrm{n}}$ value shows a relatively high value of about 1.0, and there is a tendency to have high values in $\theta=45^{\circ}$ to $90^{\circ}$. Although the $r$-value shows in-plane anisotropy similar to the $M_{\mathrm{n}}$ value, the average $r$-value is considerably higher than those of aluminum alloy sheets manufactured by normal processing and exceeds 1.0 (the calculated value: 1.07 , the measured value: 1.01$)$. The higher $r$-value seems to be an effect of near $\{111\}<u v w>$ orientation. The predicted results suggest that the deep drawability is good while the bendabil- ity is not so good in this sheet. The application of this texture to inner panels of the body can be expected in the future. On the contrary, normally cold rolled and T4-treated sheets shown in Fig. 6 seem to be suitable for outer panels which requires hemming.

\section{Conclusions}

Based on the average Taylor factor of polycrystalline face-centered cubic metals calculated by using ODF, it was possible to predict the $M_{\mathrm{n}}$ value for bendability estimation and the $r$-value for deep drawability estimation simultane- 


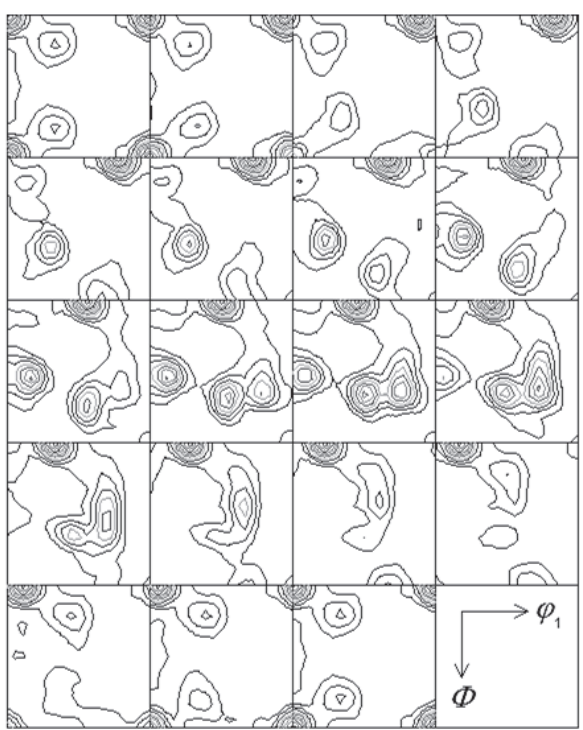

( a )

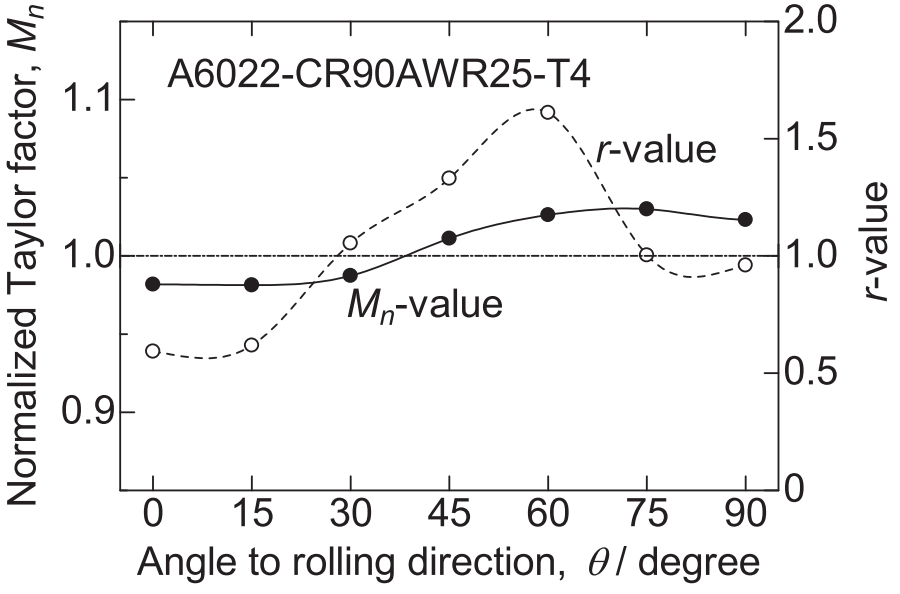

( b )

Fig. 7 (a) Orientation distribution function (ODF) with maximum orientation density of 11.2 for 6022 aluminum alloy sheet with T4 temper after $90 \%$ cold rolling and $25 \%$ asymmetric warm rolling (Contour levels: 1.0 to 11.0 at a step of 1.0), and (b) in-plane variations of normalized Taylor factor and $r$-value calculated from the ODF.

ously. In terms of texture control, the main results obtained in the present study are as follows:

(1) From the analytical results using ideal orientations, it is obvious that cube orientation $\{001\}<100>$ is excellent for bendability and $\{111\}<110>$ orientation is excellent for deep drawability. It is estimated that $\{123\}<634>$ orientation ( $\mathrm{S}$ orientation) has poor bendability and the effect of the $r$-value improvement is not so much as expected.

(2) The analytical results using real textures of annealed aluminum alloy sheets suggest that cold rolling which develops cube orientation during annealing is effective for the improvement in bendability, and that the addition of asymmetric warm rolling possible to develop $\{111\}<u v w>$ components such as $\{111\}<110>$ during annealing is effective for the improvement in deep drawability.

\section{REFERENCES}

1) K. Yoshida: J. Jpn Soc. Mech. Eng. 84 (1981) 261-267.
2) H. Inoue and T. Takasugi: Mater. Trans. 48 (2007) 2014-2022.

3) H. Takeda, A. Hibino and K. Takata: Mater. Trans. 51 (2010) 614-619.

4) H. Kaneko and T. Eguchi: Mater. Trans. 53 (2012) 1847-1851.

5) H. J. Bunge: Texture Analysis in Materials Science, Translated by P. R. Morris, Butterworths, (1982).

6) H. Inoue: Mater. Trans. 56 (2015) 61-69.

7) W.T. Lankford, S.C. Snyder and J.A. Bauscher: Trans. Am. Soc. Met. 42 (1950) 1197-1232.

8) H. Inoue, M. Hori, T. Komatsubara, H. Tanaka and T. Takasugi: Mater. Sci. Forum 558-559 (2007) 207-212.

9) H. Inoue: Mater. Sci. Forum 783-786 (2014) 33-38.

10) H. Inoue: J. Japan Inst. Light Metals 65 (2015) 156-163.

11) H. Inoue and N. Inakazu: J. Japan Inst. Light Metals 44 (1994) 97-103.

12) H. Inoue, T. Yamasaki, G. Gottstein, P. Van Houtte and T. Takasugi: Mater. Sci. Forum 495-497 (2005) 573-578.

13) H. Inoue, Y. Yamada, S. Suzuki and H. Morita: Report of the Sectional Meeting on Textures in the Japan Institute Light Metals No.56, Controlling and Formation Mechanism of Texture, (2011) pp. 1-26.

14) M. Kuroda and V. Tvergaard: Int. J. Plast. 23 (2007) 244-272.

15) L.G. Schulz: J. Appl. Phys. 20 (1949) 1030-1033.

16) H. Inoue and N. Inakazu: J. Jpn. Inst. Metals 58 (1994) 892-898.

17) H. Inoue and T. Takasugi: Z. Metallk. 92 (2001) 82-88.

18) H. Inoue: Materia Japan 40 (2001) 589-591. 